\title{
Time: An Emergent Property of Matter
}

\author{
Dirk J. Pons ${ }^{1}$, Arion D. Pons ${ }^{1} \&$ Aiden J. Pons ${ }^{2}$ \\ ${ }^{1}$ Department of Mechanical Engineering, University of Canterbury, Christchurch, New Zealand \\ ${ }^{2}$ Rangiora New Life School, Rangiora, New Zealand \\ Correspondence: Dirk J. Pons, Department of Mechanical Engineering, University of Canterbury, Private Bag \\ 4800, Christchurch 8020, New Zealand. E-mail: dirk.pons@canterbury.ac.nz
}

Received: August 21, 2013 Accepted: September 10, $2013 \quad$ Online Published: October 14, 2013

doi:10.5539/apr.v5n6p23 URL: http://dx.doi.org/10.5539/apr.v5n6p23

\begin{abstract}
A non-local hidden-variable (NLHV) design called the Cordus conjecture is applied to address the ontological question: What is time? A novel multi-level concept emerges for time, and the origin of the arrow is also explained. According to this theory, time at the fundamental level consists of the frequency oscillations of matter particules, and thus time is locally generated and a property of matter. At the next level up, that of the assembly of matter particles via bonds and fields, the interconnectedness creates a patchwork of temporal cause-and-effect, and hence a coarser time. Entropy, classical mechanics, the arrow, and our perception of time are shown to all arise at the transition from coherence to decoherence. Time at the macroscopic level is therefore a series of delayed irreversible interactions (temporal ratchets) between sub-microscopic domains of matter, not a dimension that can be traversed in both directions. The theory extends to time at the level of organic life. It explains how the human-perception of time arises at the cognitive level, and why we perceive time as universal. This theory suggests that time is all of particle-based vs. spacetime, relative vs. absolute, local vs. universal, depending on the level of assembly being considered. However it is also none of those things individually.
\end{abstract}

Keywords: time, cognition, spacetime, quantum decoherence, arrow of time, irreversibility, de Broglie, frequency, time dilation, discoherence

\section{Introduction}

Though intuitively familiar, time is a mystery. Time is a variable throughout physics: classical mechanics, thermodynamics, quantum mechanics (QM), and general relativity (GR) all include it. The topic is relevant to many discourses other than pure physics. It raises questions in philosophy for how life exists in the framework of finite time, theological questions about what existence there might be beyond time and this universe, and psychological questions about how we perceive time in a cognitive sense.

All these approaches, physics, psychology, philosophy, have theories for time. Yet the constructs in each are very different, and are poorly integrated. Those of quantum mechanics and general relativity are particularly difficult to reconcile. Nor are those constructs always coherent with humans' personal cognitive perception of time. For example, the idea that time runs differently depending on gravitation or velocity, or that time may have had a beginning (and therefore not existed before the universe), is deeply puzzling to the mental model of many people. There are also unsolved integrative problems, like how the time that emerges at the level of atomic clocks transfers to the world at large, whether there is an absolute time, how time started, how time dilation works, and how the arrow of time arises. Any new solution for time needs to answer these foundational and integration problems.

This paper offers a reconceptualisation of time, using a non-local hidden-variable (NLHV) design called the Cordus theory. This has previously been used to develop an explanation for entanglement, a solution to wave-particle duality (Pons, D. J., Pons, A. D., Pons, A. M., \& Pons, A. J., 2012b), and a theory for the strong force (Pons, D. J., Pons, A. D., \& Pons, A. J., 2013). In the present paper we extend the concept to develop a theory for how time arises. This theory predicts that time is an emergent property of matter. Using we show that it is possible to give a comprehensive explanation of time, its origins, where the arrow arises, and how it scales from the fundamental level to the macroscopic world. 


\section{Literature}

There is no universal-theory of time that is acceptable to all the disciplines. The problem of time is also strongly coupled to several other foundational problems in physics, described below, so that they can be considered linked problems.

In physics, time - or rather the apparent lack thereof-is a feature of entanglement, whereby two geometrically separated particles are able to superluminally influence each other, or at least respond to measurement in a co-ordinated manner (which may not be the same thing). So this drags locality, realism, and local realism into the debate. These come with their own ambiguities of definition and debates about how to represent them mathematically. In turn entanglement suggests that either the fundamental reality is probabilistic (the Copenhagen interpretation) or that particles have an internal structure, i.e. a non-local hidden-variable (NLHV) solution, hence the Einstein-Podolsky-Rosen (EPR) criticism (Einstein, Podolsky, \& Rosen, 1935). The bulk of opinion favours a zero-dimensional point, and this is the underlying premise of quantum mechanics.

\subsection{Entropic Approaches}

The problem of time is also closely linked to entropy, and indeed the concept of "arrow-of-time" is often discussed in that context. Time features in general relativity and thus gravitation in general. It is expected to be involved in any theory of quantum gravity (Jejjala, Kavic, Minic, \& Chia-Hsiung, 2012). One of deep problems is that the interactions of fundamental particles are reversible (symmetrical). This is not contentious, as both Newtonian physics and quantum mechanics are comfortable with the idea that dynamic interactions are invariant when time is reversed (time-reversal invariance), and this is seen most clearly in equations of motion (Aiello, Castagnino, \& Lombardi, 2008). Hence the micro-reversibility principle, that past and future directions cannot be distinguished in a system at equilibrium (Campisi \& Hanggi, 2011).

The question is how the irreversibility of macroscopic behaviour emerges from the reversible physics. This is the problem of asymmetrical-time. This time-asymmetry is a large and persistent problem ("the ever-lasting question", Campisi \& Hanggi, 2011), and although many ideas have been put forward, there is no completely satisfactory solution. The basic idea is that the arrow of time is associated with irreversibility, hence also entropy and the Second law of thermodynamics. Specifically that time is related to the entropy gradient.

Much effort has been exerted to explain how and where the irreversibility arises, with an expectation that this will yield an explanation of time too. Any number of entropic solutions are on offer with various features. For example: time is a correlation device relative to a clock (master clock) to "keep track" of the sequence of instants or small changes arising at the quantum level (Caticha, 2011); time emerges from the irreversibility of nonlinear interactions and phase changes within complex systems (Mainzer, 2010). However that complexity argument is contentious. The question, which is also relevant to chaos theory, is whether complexity necessarily results in irreversibility, not at all, or only at certain thresholds. Also, even if it does provide irreversibility, whether it still preserves determinism.

The difficulty is compounded by not knowing whether the arrow of time exists at every scale from the fundamental particle to the universe, or emerges at some particular scale. A complementary idea is that there may be several arrows of time (Penrose, 1979). However the residual question with this approach remains: where (at what level?) and how does the irreversibility of time arise?

\subsection{Spacetime}

While one line of attack on the problem, perhaps the main approach, has been to associate time and entropy, there have also been other approaches. One of these is to construct time as non-entropic and instead as an intrinsic property of spacetime (Aiello, Castagnino, \& Lombardi, 2008). This is because entropy has problems of its own. It is defined well-enough in engineering, but its definition at the level of the cosmos is still controversial (Aiello, Castagnino, \& Lombardi, 2008). However, the mechanisms are unknown whereby time might be such a property of spacetime. In addition, there is dissention as to whether spacetime has a substantial status or is merely the relationships between bodies (Lyre, 2008).

\subsection{Philosophy}

Philosophical issues abound (Lyre, 2008). There is the issue of the Now: how the future becomes the present human experience of reality, and then immediately becomes the irrevocable past. This also connects to theological issues about predestination and free-will (Hasker, 2011) (Note 1). Then there is the question of the existence and permanence of three-dimensional objects in time. Related to that is the question of how measurement by an observer changes the system, and whether spacetime is an infinitely divisible continuum, hence also the Zeno paradoxes (Note 2). 
Others perceive the whole problem is simply an artefact of an anthropocentric perspective; that the difference between past and future is a perception and that it is illegitimate to word the problem of the arrow-of-time in these terms (Aiello, Castagnino, \& Lombardi, 2008). To this line of thinking, temporal asymmetry is a merely a cognitive artefact, and we are to view the problem from outside time (atemporal). However this is ontologically unsatisfactory because no explanation is available for the cognitive mechanisms, nor for how the proposed underlying temporal symmetry would operate, nor for the atemporal state. There are too many unsubstantiated premises. In some theology perspectives God is atemporal (Hasker, 2011).

To summarise, there is a rich tapestry of concepts about time, yet no big picture. There are many competing theories, and selecting between them is a matter of personal choice rather than any preponderance of evidence.

\section{Purpose and Approach}

\subsection{Purpose}

The purpose of this paper is to develop a theory for time, starting from a specific NLHV perspective called the Cordus theory. This is worth attempting because the NLHV sector has previously delivered novel insights into fundamental processes. It may be possible to unify the different perspectives, and perhaps even access a deeper concept for time.

\subsection{Method}

We formulate the research question as a conceptual one, as opposed to one of quantitative mathematical modelling, and select our method accordingly. Specifically, we start by drawing logical inferences from the Cordus theory, to create a theory for the fabric. We then apply design thinking to identify what mechanics at the particule level would be sufficient to explain time phenomena. To achieve this, we use a systems engineering design method. This involves taking the functional requirements (observed temporal behaviour of matter) and inferring the requisite attributes (internal and external mechanics of the particule).

\subsection{Approach}

Our approach is as follows. Accepting that time dilation does occur, and accepting also that atomic clocks do show a physical representation of that effect (as opposed to some other effect), then it is a reasonable assumption that at least the ticks of time are really represented in the mechanism of the clock. Although that mechanism is uncertain, we accept that it involves transient atomic behaviour, and therefore the ticks of time are associated with atomic events. A continuation of this line of thinking and the application of design principles, leads to a novel theory for time.

We start with the existing ideas of the Cordus theory. The Cordus conjecture postulates that all particles are one dimensional structures of finite length, and emit three-dimensional discrete forces at their two ends. This is called a particule. It is a NLHV design. It has previously been used to explain entanglement and wave-particle duality (Pons et al., 2012b). Second, we extended the theory to explain frequency, and this explains the frequency of photons, and the de Broglie frequency of matter (Pons, D. J., Pons, A. D., Pons, A. M., \& Pons, A. J., 2011d). Third, we developed the theory to include discrete forces and the corresponding discrete field elements. This explains the electro-magneto-gravitational (EMG) forces, and the strong interaction (Pons et al., 2013). Frequency is then the rate at which a particule is able to interact with other particules - this becomes an important concept later in the time theory. Third, we then created a theoretical construct to differentiate between coherent and decoherent states of matter (Pons, 2012a).

Putting it all together, we show that a logically consistent explanation is that time arises as the frequency cycles of a NLHV particule, and consequently that time depends on how the particules are assembled into matter and hence physical bodies. The present paper reports on this time concept and its implications at fundamental and macroscopic levels. The theory has some unexpected implications and makes some falsifiable predictions, which are discussed.

\section{Results}

The results of the design effort suggest that if time were to be constructed as follows, then it is possible to accommodate all the existing perspectives and also answer several of the troublesome ontological questions about time. The results suggest there is a hierarchy of time, with the physical size of the body being the main scaling factor, and we present the results in this order, starting at the fundamental level and building up to the macroscopic level and onwards to the human perception of time.

In understanding this theory, it is important to recall the basic features of the Cordus particule, and as an aide-mémoire the electron is shown in Figure 1. For a fuller discussion see (Pons et al., 2013). 


\section{Electron e}

Characterised by one discrete force (vis) in each of the three directions. Therefore a highly stable structure. Together with anti-electron is manufactured from photons (pair production)

The vires discrete forces are released rather than retain as in the photon. Consequently there is an enduring hyff in each of the three directions, which creates a long-ranged force effect.

New vires discrete force elements continue to be created and sent down the hyff at each frequency cycle

Each discrete force element carries a $1 / 3$ electrical charge, with the sign representing the direction, so electron has overall -1 charge.

Fibril, provides instantaneous communication between reactive ends

Type of reactive end:pulsatile. One reactive end energising and the other de-energising $\left(180^{\circ}\right.$ out of phase)

The HED notation is a Cordus symbolic representation of the distribution of the discrete forces in the three emission directions (HEDs)

\section{HED notation}

Three orthogonal axes $(r, a, t)$ for emission of discrete forces

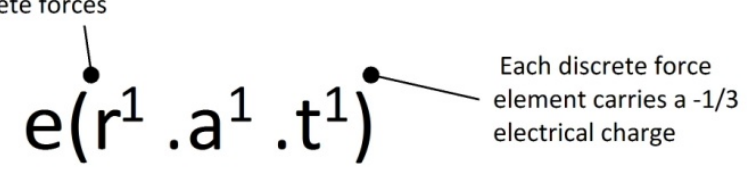

Figure 1. Cordus model of the electron. It is proposed that the particule has three orthogonal discrete forces, energised in turn at each reactive end

\subsection{Fundamental Time: Frequency Oscillation of the Particule}

The fundamental tick of time, in the Cordus theory, is the frequency of the particule, which is identified as the cycle of re-energisation of the two reactive ends. Cordus provides a specific internal structure for particules, hence a physical explanation for frequency (Pons et al., 2011d). Thus fundamental time becomes:

Fundamental Time, at the level of the individual particule (e.g. electron) (level 1), is the frequency of the re-energisation cycles of its two reactive ends.

There is a fundamental tick of time for each particule, and this affirms the QM perspective of time. Particules with greater masses or energies have higher frequencies $(E=h f)$, and therefore tick faster. Thus there is no universal time in this Cordus theory, but rather a unique time for each particule. These relationships are summarised in Figure 2, which shows the functional situation in the system modelling representation of integration definition zero (IDEF0) (FIPS, 1993). 


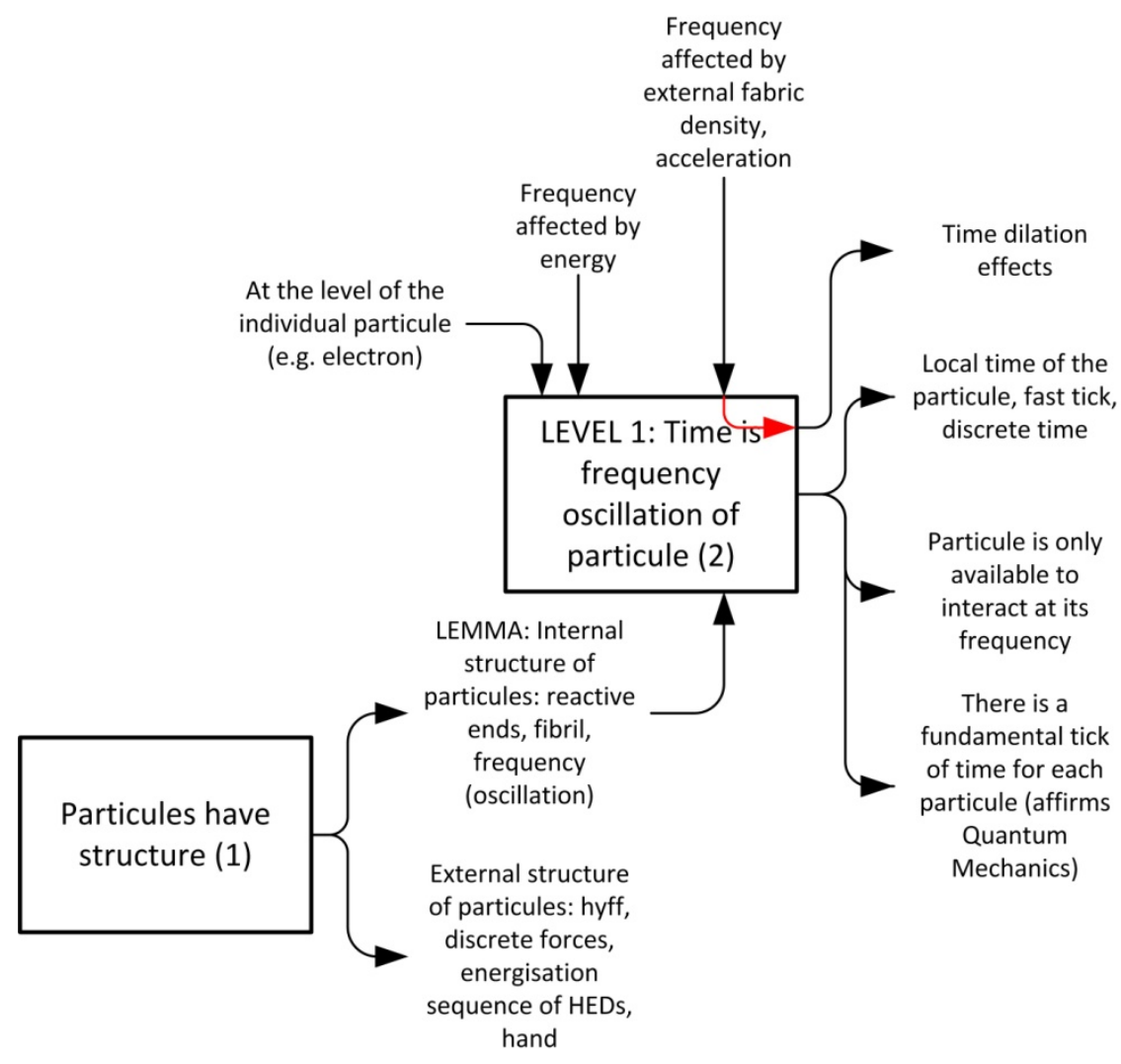

Figure 2. The Level 1 Cordus theory for time is that the cycle of re-energisation of the two reactive ends, at the frequency of the particule, comprises the fundamental tick of time. At this level time is reversible. The diagram represents the causality whereby particule-structure and external events affect the tick of time for that particule

Linking time to the re-energisation cycles is important because the particule is only available to interact with other particules when it is energised (Note 3). When a reactive end of particule A is energised it issues discrete forces. These are propagated outwards at local fabric speed $\mathrm{c}$, the speed of light. The discrete force carries the electro-magneto-gravitational field, which therefore is also discrete, and also carries the strong and bonding forces. These fields \& forces inform neighbouring particules B, even remote ones, about the state of the basal particule A. So remote particule B moves in response to the fields from A. Likewise B emits its own fields, and some of these are intercepted by A. Thus particule A responds to fields \& forces from the external environment, and this response occurs when its reactive ends energise. Thus the periodic re-energisation of the reactive ends is a mechanism whereby particules communicate with other particules and respond to their forces (Note 4).

In this theory time is therefore fundamentally an attribute of matter, particularly its frequency, as opposed to being an external dimension or imposed variable. Every particule transmits its time signature into its surrounds, in the form of its discrete field elements, and is only available to interact with other particules then.

\subsection{Assembly Time}

The Cordus theory suggests a particular multi-level interpretation for time. In this construct, time at the fundamental level is generated by each individual particule, and is associated with the frequency of the particule. Of the different times within the Cordus theory, this ticks the fastest. However, particules will generally not have identical frequencies, and even like particules with different energy or in different situations will tick differently. Also, particules are more commonly, and more usefully from our perspective as inhabitants of the universe, bonded together in atoms, molecules, crystals, organelles, and living bodies. In the Cordus theory we refer to this as "assembly".

\subsubsection{Time Depends on the Level of Assembly of Matter}

Therefore if time fundamentally exists at the level of the individual particule, then time also exists-in some way - at the level where those particules are assembled. However, since time is determined by interaction between particules, the nature of time will logically depend on the nature of the assembly. This interaction occurs 
since each particule emits discrete forces, and these interact with neighbouring particules, either strongly as in bonding, or weakly as in macroscopic fields. Thus the next lemma in the Cordus theory is that time depends on the level of assembly:

Time (level 2) depends on the level at which the assembly of matter occurs, being dependent on the nature of the interaction between the assembled particules, and hence dependent on the nature of the bonding.

Next, we explore the different ways that particules can be assembled, namely coherent and decoherent bonding, a mix of the two, and remote fields. We take the simpler case of coherent matter first, and provide physical explanations for these states using the Cordus conjecture.

\subsubsection{Time at the Level of Coherent Matter (Level 2.1)}

The Cordus theory explains coherence as multiple particules being in complementary geometric locations and frequency states (Pons, D. J., Pons, A. D., Pons, A. M., \& Pons, A. J., 2011f) due to the synchronous interaction (Pons et al., 2013). In other words, a particule, which has two ends, shares the locations of its reactive ends with those of other particules. Importantly, frequency is also involved: all the particules must have a common (or harmonic) frequency. This arises from the strong force, which in the Cordus theory is recast into the synchronous interaction (Pons et al., 2013). This, as the name suggests, arises from neighbouring particules synchronising their emission of discrete forces. This causes the participating particules to be interlocked in geometry, frequency, and phase. The interaction pulls or repels particules into co-location and then holds them there, hence the apparent attractive-repulsive nature of that force and its short range. Those discrete forces are renewed at the de Broglie frequency of the particule. The Cordus theory goes further, by proposing that the synchronous interaction is intimately linked to coherence, with the electro-magneto-gravitation (EMG) forces being the associated decoherent phenomenon. See Appendix A for details.

In the case of entanglement, which was the specific situation under examination in the EPR paradox (Einstein, Podolsky, \& Rosen, 1935) and the resulting Bell-type criticisms (Bell, 1964; Leggett, 2003), the Cordus theory offers the following explanation: that photons A and B are particules that have, through the creation of a coherent light source, become locked together and synchronised in a complementary manner through their discrete field structures, such that changes to the one affect the other. The photon spans may be subsequently stretched so that the reactive ends are far apart. What looks like one complete photon at each site is, according to this version of events, two half photons. The fibrils of each retain their ability to communicate instantly. Changing one reactive end at one site therefore immediately changes the other reactive end of the same particule. The synchronous interlock also causes the second particule to be changed and in a way that is complementary to the first, and that change can be immediately observed at the other site. In this way the Cordus theory explains entanglement and the empirical observation that the results of the two detectors are correlated (Pons, D. J. \& Pons, A. D., 2012).

At suitably small scales all matter becomes individually self-coherent. This is why quantum mechanics, which presupposes coherence, is applicable at this level. However the difficulty is that macroscopic objects are decoherent, to the consternation of QM, and it is not clear where or why the boundary exists. The Cordus theory predicts this boundary is at or below the molecular level (Pons, 2012a). Thus electrons, protons, and atoms are always internally coherent, that being a necessity for their stability. Larger assemblies of matter may also be coherent in special cases, e.g. superfluids and superconductors. In these cases the particules form paired or network structures, hence domains. In this context a domain is a volume of massy particules, and it may be within another body or its own body. The point is that a decoherent body may, according to the Cordus theory, contain domains that are coherent.

What this means is that a coherence body synchronised frequencies throughout, and this has implications for how time ticks within these bodies. Thus we anticipate another form of time for coherent bodies, and this is similar to fundamental time but on a wider scale:

Coherent assembly time is the common frequency cycle of re-energisation of the particules within a coherent domain of matter.

For a coherent body, e.g. superfluid, the Cordus theory predicts that the whole body has one synchronised time frequency (all the particules beat together), and that frequency does not depend on the number of particules in the assembly. Also, the phase of the particules will also be complementary. These specific time-characteristics may be testable and falsified.

This functional situation is shown in Figure 3. 


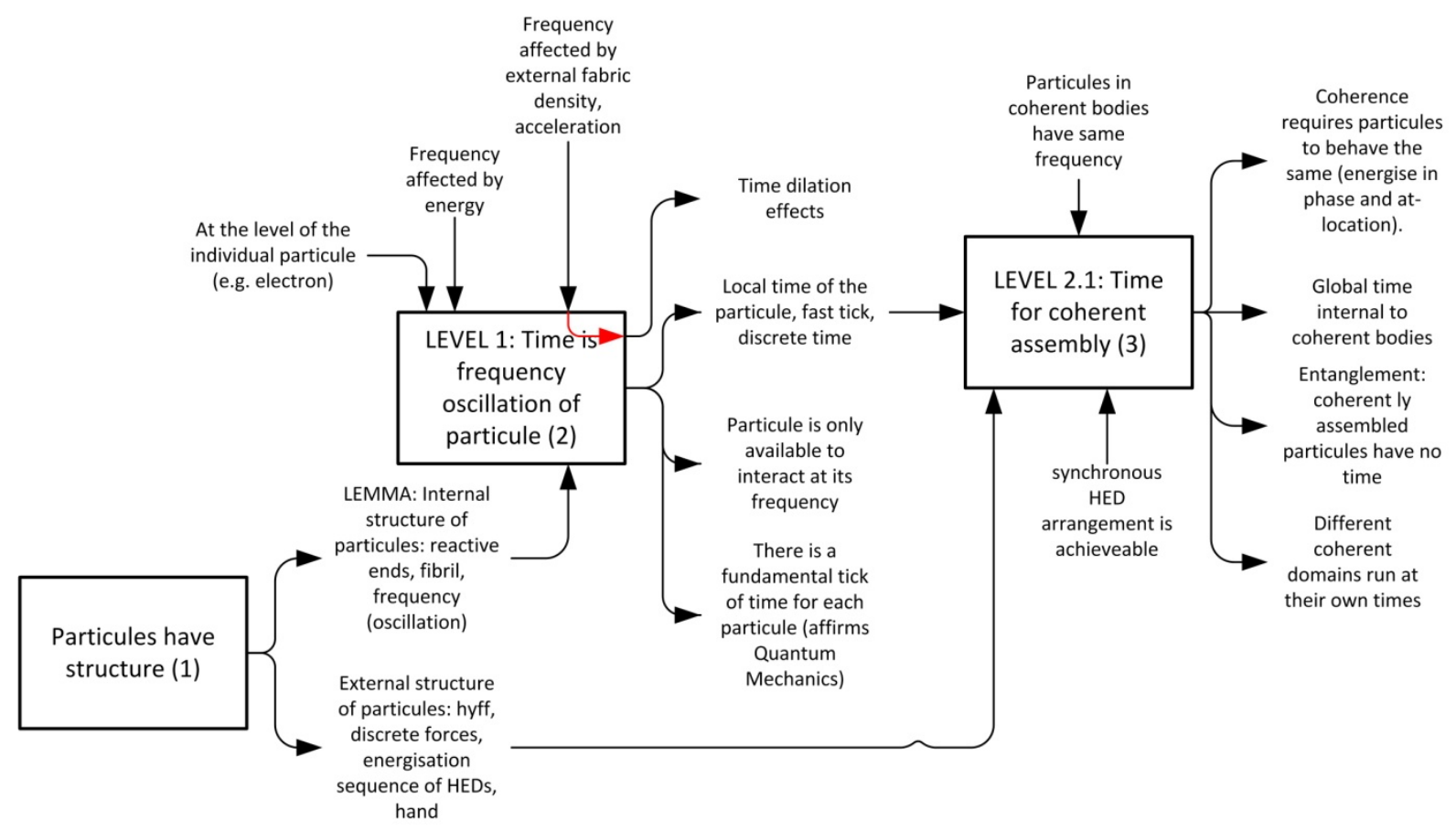

Figure 3. The Level 2.1 Cordus theory for time in COHERENT assemblies of matter

The Cordus theory thus affirms the QM concept of global time, but only for coherent bodies.

At this level the arrow of time does not exist in principle, though it may in practice. This is because a coherent body can have all its internal particules put back in their original place, i.e. change is reversible. Of course this requires that we accept that identical particules are indistinguishable and can substitute for each other, which seems reasonable. This might be possible in simple systems of only a few coherent subatomic entities, in prescribed states, and a stable external environment. If the particules can only be in a few states, then their behaviour is effectively reversible. (If an assembly only has a few unique states, i.e. positions that its atoms can be in, then the chance of reverting to that state is higher, i.e. a type of Markov process.) There is still interaction at frequency cycles, i.e. time, but it no longer has an arrow pointing away from past states. So the Cordus theory suggests that time and the arrow-of-time are not synonymous at all levels. The self-stability of the proton is an example: it experiences time, but there is no arrow of time forcing it into a different state.

However it is impossible to fully control the external environment of the fabric (the cumulative effect of all the fields created by all the other particules in the observable universe) and the perturbations it imposes on the body under examination. Note that even in the simplest situation of two coherent interacting domains, there is still the perturbation of the fabric discrete forces that they both feel, i.e. the rest of the particules in all the accessible universe affect the two domains. If these external forces are strong enough, they create additional geometric positions for the system to move into. As soon as the system moves into one of these, it will then have additional states available, resulting in a decreased chance of returning to the original state. Furthermore, by moving into a new position, the system's contribution of discrete forces back into the universe also changes. So the system also changes the universe, hence further difficulty getting everything back into its original state. The decay of the free neutron is interpreted in Cordus as an example of a stable case slipping into decoherence under external disturbance (Pons, 2011a).

\subsubsection{Time at the Level of Decoherent Matter (Level 2.2)}

Macroscopic objects at our level of existence are decoherent as a whole, which the Cordus theory explains as caused by the homogeneity of composition and being too warm to be coherent (Pons, 2012a). We suggest there is an assembly tree to any macroscopic object, where the sub-components may be a mixture of individually coherent and decoherent domains. However as the assembly grows in size and diversity of composition, so the synchronous interaction of discrete forces (hence coherence) becomes impossible to negotiate by the protagonist particules, and decoherence arises. For example, even if individual molecules are indeed coherent then an aggregation of different molecules can be decoherent as a whole. For coherent and decoherent domains, see 
Figure 4.

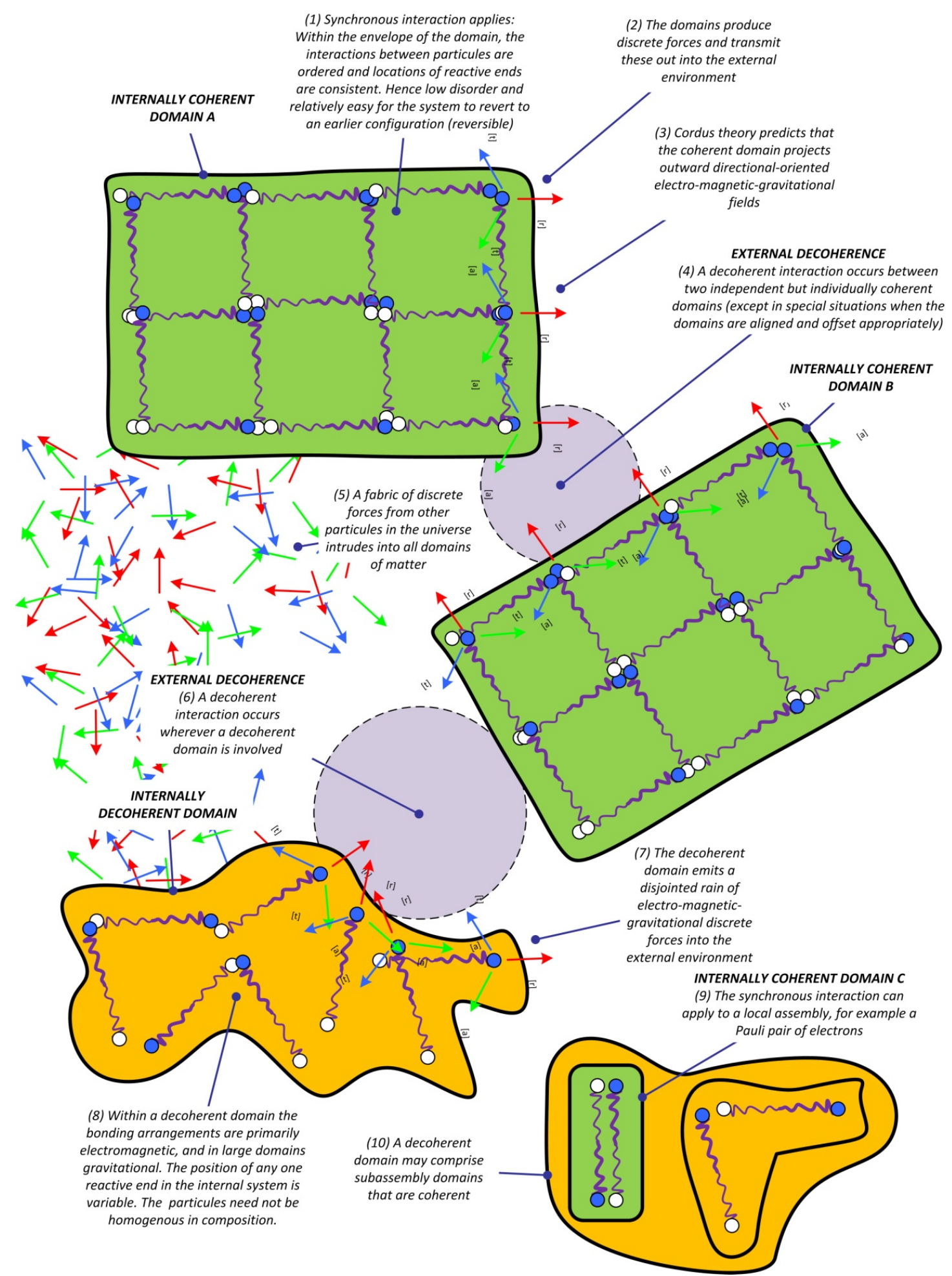

Figure 4. Representation of coherent and decoherent domains of matter, and their various types of interaction. Coherence is a state of coordinated positional relationships within a domain. However even these bodies are subject to perturbation by the discrete forces of the fabric. Note that the relationship between coherent bodies is not necessarily or even typically coherent. Decoherent domains, which may contain coherent subassemblies, always have decoherent external relationships 
Single particules are automatically coherent. These, and any coherent domains, externally manifest their properties at their own internal frequency. These properties are their fields (electrical, magnetism, and gravitation), the orientation thereof, and the two positions of the reactive ends. The fields themselves comprise discrete forces, and the frequency of production is very high. However other neighbouring domains of matter of different composition, even if independently coherent, do not perceive the individual discrete forces of the first domain in their discrete synchronous form (Note 5). Instead they perceive each other (experience each other's forces) as a disjointed rain of field forces. Thus we anticipate a different type of interaction between particules in decoherent bodies, and we interpret this as another, and slower, level of time:

Decoherent assembly time is the tick of interactions within an aggregate body of coherent and decoherent sub-bodies. This tick of time is much slower than the fundamental time, and derives from individual particules responding as they can to the rain of field forces.

This in an important concept since we go on to propose that this type of time dominates organic chemistry, and hence also our human perception of time. The concept is also important as it explains why the irreversibility of time occurs, as described next.

\subsubsection{Arrow of Time}

Decoherence causes a time delay to be inserted into the functional interaction of two or more domains - whether or not those domains are individually coherent. This because the frequencies differ, so the faster oscillating domain will have to mark more ticks before the slower responds. Since there is geometric separation, however small, then the finite speed of field propagation (c, speed of light) adds a further time delay. Consequently the one domain generally has done something different, e.g. responded to a third domain, or emitted some discrete forces into the universe, before the second has fully responded. Therefore getting domains back into their initial positions becomes unlikely and statistically impossible as the number of participating domains increases. So what happens stays happened, and does not naturally self-repair. We sum this up as follows:

Decoherent assembly time is irreversible, hence the arrow of time arises at this level. This is because the interaction between subassemblies is practically irreversible due to intervening changes, propagation delays, and the complexity of large number of participating particules. This is also where and why entropy arises.

Hence we propose that classical mechanics and decoherence arise at the same point in the assembly tree of matter. The macroscopic perception of time arises at the same point, as does entropy. This logic is represented in Figure 5.

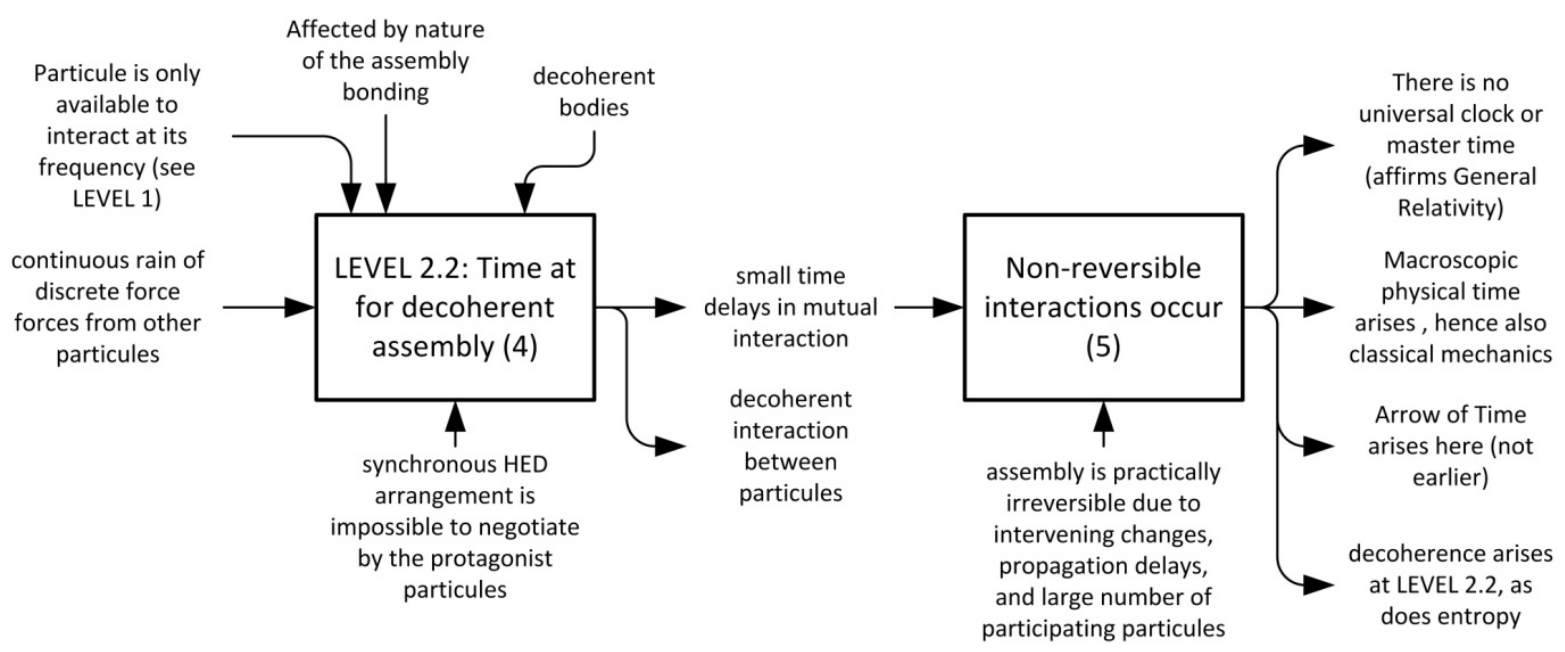

Figure 5. The Level 2.2 Cordus theory for time in DECOHERENT assemblies of matter

While reversibility seems feasible at simple levels, we never see this for macroscopic bodies. We suggest this is because such bodies are decoherent. More accurately, their relationship with their external environment is decoherent even if their internal bonding arrangements are coherent. Thus we note a derived lemma:

Macroscopic bodies invariably have decoherent relationships between them. Such bodies, regardless of 
whether they are internally governed by coherent or decoherent relationships (bonds), interact in-elastically with their environment, in that such bodies do not return to precisely their initial states. Inability for one body to return thereby means that all the other bodies in the accessible universe cannot either, because the fabric of background field (discrete forces) has been changed.

\subsubsection{Long-Range Interactions through the Fabric}

Up to here we have been examining particules that are in some close assembly relationship due to bonding. However there is also the case of bodies that interact with each other at a distance, through the electro-magneto-gravitational (EMG) forces. To help solve this we apply principles from the existing Cordus theory for unification of the forces (Pons, D. J., Pons, A. D., Pons, A. M., \& Pons, A. J., 2011b; Pons, D. J., Pons, A. D., Pons, A. M., \& Pons, A. J., 2011e) and the content of the vacuum (Pons, D. J., Pons, A. D., Pons, A. M., \& Pons, A. J., 2011c).

Any one particule A receives discrete field discrete forces from all the particules (many Bs) in its accessible universe (past light cone). Space within the universe is therefore filled with a skein of discrete fields in transit, which in the Cordus theory is termed the fabric (Pons et al., 2011c) (Note 6). Thus the Cordus time theory predicts there is another variant of time, at the level of macroscopic bodies subject to EMG interactions, and we call this the fabric time. It is a subtype of decoherent assembly time:

Fabric time is the mutual interconnectedness of matter particules spread over three-dimensional space. This occurs via the fabric, comprising discrete field forces for electric-magneto-gravitational interaction. This is a coordination of events across space.

In this theory the fabric, and the EMG fields it carries, causes a connectedness between particules. They respond together, even if in a slightly delayed manner as their separation increases. There is therefore a consistency and smoothness to the interaction between particules, mediated by the fabric. The resulting interaction stitches together three-dimensional domains of space (matter and vacuum-fabric) into a macroscopic collated time. This level of time passes more slowly, due to the many tiny delays required for particules to react to each other, given the dissimilar-frequency and phase-differences between the particules. There is no real tick at this level, but rather a one-directional mutual causality. This, the Cordus theory suggests, is where the arrow-of-time arises, and what general relativity perceives as spacetime. This is also the macroscopic level of physical time, and hence where our human perception of time first arises.

This Cordus concept of 3D fabric affirms the general relativity perspective of spacetime. It also provides an ontological answer to one of the earlier questions (Lyre, 2008): it suggests that spacetime has a quasi-substantial status (comprises discrete forces) but has no universal time-signature per se, and mainly represents merely the relationships between bodies.

According to this theory it is important to differentiate between the relationships within and between domains when considering coherence. This principle is illustrated in Figure 4.

\subsubsection{Time-Dilation Explained}

Furthermore, the Cordus theory of time provides a mechanism whereby the external environment can push back in and affect the frequency of the particule, and thus an explanation readily arises for time-dilation. The proposed mechanism is as follows. An encounter with greater fabric density causes the frequency of a particule to slow down, hence time runs slower. This is because the high density of external discrete forces makes it difficult for the particule to emit its own discrete forces $>$ emission is retarded $>$ energisation of reactive end is delayed $>$ frequency lengthens.

It is known from general relativity that a body experiences time dilation in any of the following three situations: higher relativistic velocity, or higher acceleration, or in a high gravitation field. According to the Cordus theory, all these are situations of greater fabric density: the first because the fast-moving particule is at a speed approaching that of the fabric itself and therefore emission of the particule's discrete forces is resisted (from the perspective of the particule, the external discrete force fabric is saturated), the second because the accelerating particule emits discrete forces which it then moves into, thus creating its own locally high fabric density, and the third because high gravitation field is intrinsically a high external fabric density. (Note 7)

\subsection{Organic-Life Time: The Speed at Which Chemical Interactions Occur in Decoherent Bodies}

Having differentiated between the different types of time at different assembly levels, and having identified how time works for decoherent bodies, we are now ready to extend the theory to higher levels, including living creatures. This is now the third level of time. 
Our own physical bodies consist of cells and tissues which are decoherent domains at any level which our unaided senses can perceive. Of course at some finer level even the decoherent domains comprise coherent particules, but the point is that such assemblies of matter operate with decoherent assembly time. We anticipate that the only coherent domains within physical bodies are at the molecular level and smaller, possibly as large as organelles (Pons, 2012a). The actions of the cell are not superluminal, but some molecular interactions might be. Thus the Cordus theory states that there is a slower level of time which is based on the speed at which chemical reactions can take place, and this governs the physiology of organic life:

Organic-Life Time, at the level of an individual cell, consists of the nebulous aggregation of the discrete fields of the many individual coherent particules (electrons, atoms, molecules) and decoherent sub-components (clumps of molecules, organelles). "Nebulous" because the discrete forces are not individually distinct. Chemical transport within the cell occurs as and when the sub-components are able to interact. Thus the cell takes much longer to achieve anything (more frequency ticks of its atoms) than a simple sum of the times required by the coherent subcomponents.

The process of human thought takes time. The photosensitive chemicals in the retina need frequency cycles to react to incoming photons, the electrons need frequency cycles to transit down the nerve fibre into the cortex, the neurotransmitter molecules need more frequency cycles to interact with other cells. Thus it takes time for the brain to assign a meaning to what is seen. At the level of organic life, time is based in chemistry. We represent these relationships in Figure 6.

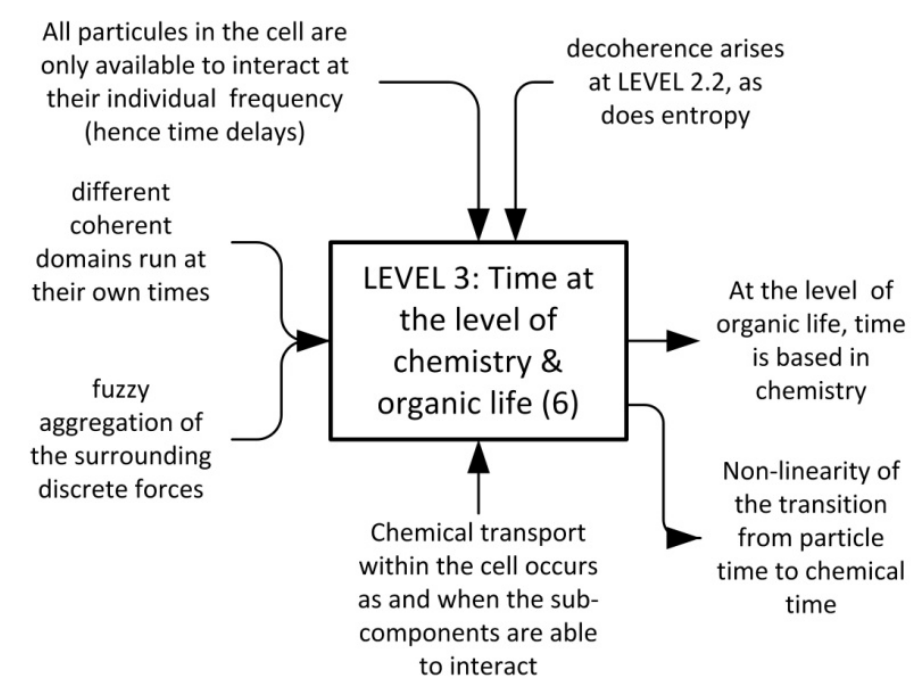

Figure 6. The Level 3 Cordus theory for time in organic chemistry

\subsection{Cognitive Time: Human Perception of Time Phenomena}

Our human perception of time is the next level up, the fourth level of time, and the Cordus theory suggests it is a construct of the cognition, built on the time of organic chemistry. The irreversibility of cause-and-effect creates a physical arrow of time. This is not merely a cognitive perception, but a real physical flow. The brain then constructs a cognitive meaning for the one-wayness of time. Proprioception, and the underlying neural systems that support it, creates the personal arrow of time. We think, then our limbs move, then our peripheral nerves confirm the new position, likewise the eyes confirm and calibrate the proprioception. To the cognitive system, the arrow of time is the immediate and predictable sequence of cause-and-effect in the neuro-musculo-skeletal system and the immediate surrounding environment.

According to this theory, the brain need not have a global atomic/molecular clock. It only needs a subjective counter of events from which to infer time (Note 8). Our cognitive quantification of time is very rough, and varies with the situation. Nonetheless we perceive time as flowing. This is because it does indeed take chemical time for us to accomplish anything, even thought, and especially motion. Also, those interactions are all irreversible, so there is no going back. The human perception of time is therefore a cognitive construct that we overlay on chemical time, and that in turn on the frequency of matter. We represent this in Figure 7. 


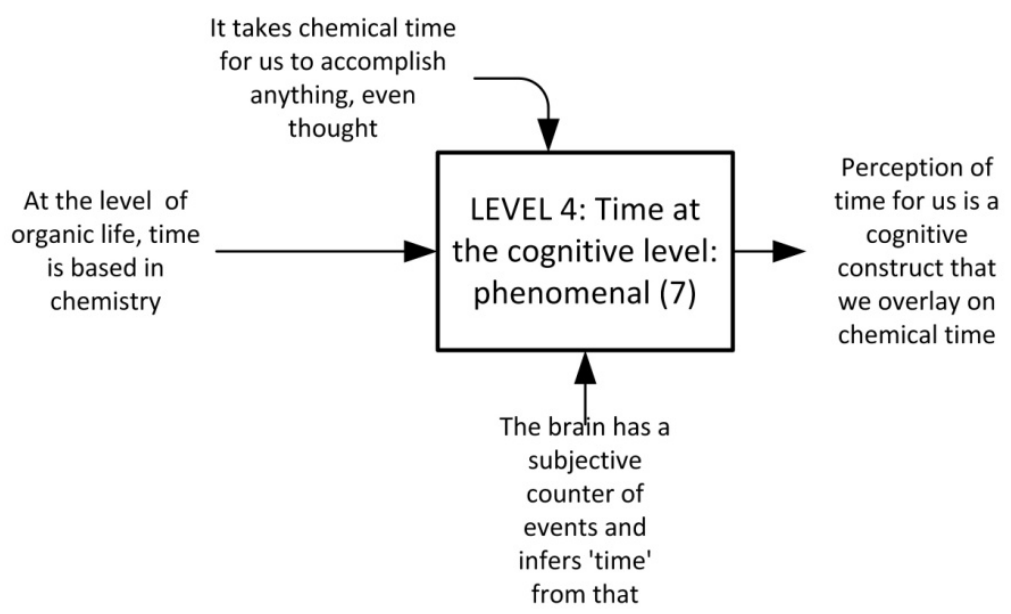

Figure 7. The Level 4 Cordus theory for the perception of time

We might perceive our thoughts to be effortless and instantaneous, and the resulting movement of our body to be immediate. We can perceive, and respond within, tenths of a second. But the deeper clocks of the particules of matter beat so fast as to be beyond our sensation. We also perceive that time flows in one direction: forward. There is an obvious arrow of time, whereby cause precedes effect. This too arises from the non-linearity of the transition from fundamental time to chemical time. We also perceive that time is universal: that what happens to me is also how you see things happening. So when we meet and I extend my hand and voice a greeting, I believe that you too hear those words, and the touch of the hands is real. Clearly this is the case (except in the most extreme philosophical interpretations), because when meeting we do indeed see the smile and confirmatory signs that we expect. To sum up:

Cognitive time, or the human perception of time, is a cognitive construct of the order in which we experience phenomena, based in turn on a neural representation of chemical time, and the arrow of time (irreversibility), both of which arise at a deeper level. The coordination provided by the fabric ensures that there is a consistency of experience between two people, hence personal interaction is a real common experience, and time is perceived as universal.

Cognitively we struggle to interpret events when the sensory signals conflict, like echoes in a large room, or time-delay in a long-distance call. The fact that the cognition struggles in such cases is circumstantial evidence of a cognitive model for the arrow of time. Worse, if one person exists at a faster (or slower) pace of time, as in time-dilation, then the cognitive model struggles and we perceive the situation as bizarre. That our feet age slightly differently to our head is only strange because we expect, cognitively, that time be continuous and universal, as is usually our experience.

Philosophical Considerations

With the irreversibility theory given here, we can explain why there is a past, and with the fabric theory we can explain why there is a unique present (Note 9). Thus the universe only need have a single possible past. Also, the present moment is a consequence of that single past, and will evolve into a single future, i.e. there need be only one universe that we all share. Regarding the philosophical question of what is the NOW (the present moment), we suggest it is a cognitive effect associated with consciousness, memory, and the process of thought. Another important consideration is the Cordus concept of how the fabric represents the forces of all the other particules in the accessible universe, and how the response of the recipient particule then changes its contribution to that fabric, and therefore changes the universe - or at least makes the universe irreversible. Thus the NOW is a summary of the many mutual interactions between particules throughout the universe, and there need only be one NOW that we all share and influence.

\subsection{The Connectedness of Time}

Finally, we examine the question of how multiple bodies interact, and how the coordination arises. We have already identified that there is no master clock, but if that is lacking then we still need a coordination mechanism. There is a connectedness of phenomena that are at different geometric locations. It seems that spacetime is continuous, because it seems that it is possible to coordinate the two phenomena in time. We show that the two 
phenomena are linked, because they share the same fabric.

Any communication between two objects is a result of photons, or massy particules, or fields, and these cause positional constraints on the other, i.e. the geometric location of the reactive end is affected by the communication. Thus all force is ultimately prescribed displacement of position of the target particule. A phenomenon that occurs in one volume of matter, be that combustion, noise, motion, etc., thereby communicates that to other matter around it. Consider one volume to be my body: my speaking transmits forces to the volume of air immediately around me, which in turn propagates the dynamic displacement throughout its bulk, so that the membrane in your ear is displaced, and you hear the sound. In general the phenomenon is that one volume of matter causes an effect in the second. The interactions at the most basic level all require frequency cycles, so this causes temporal causality. This is a physical reality, and is also the basis for cognitive perceptions of time. Thus we infer:

It is not a master clock that accomplishes the temporal connectedness of phenomena that are at different geometric locations, nor does it require continuity of spacetime per se. The piece-wise communication, via discrete field interactions of the fabric, between adjacent volumes of space (matter and fabric) applies spatial consistency to time.

This causality is represented in Figure 8.

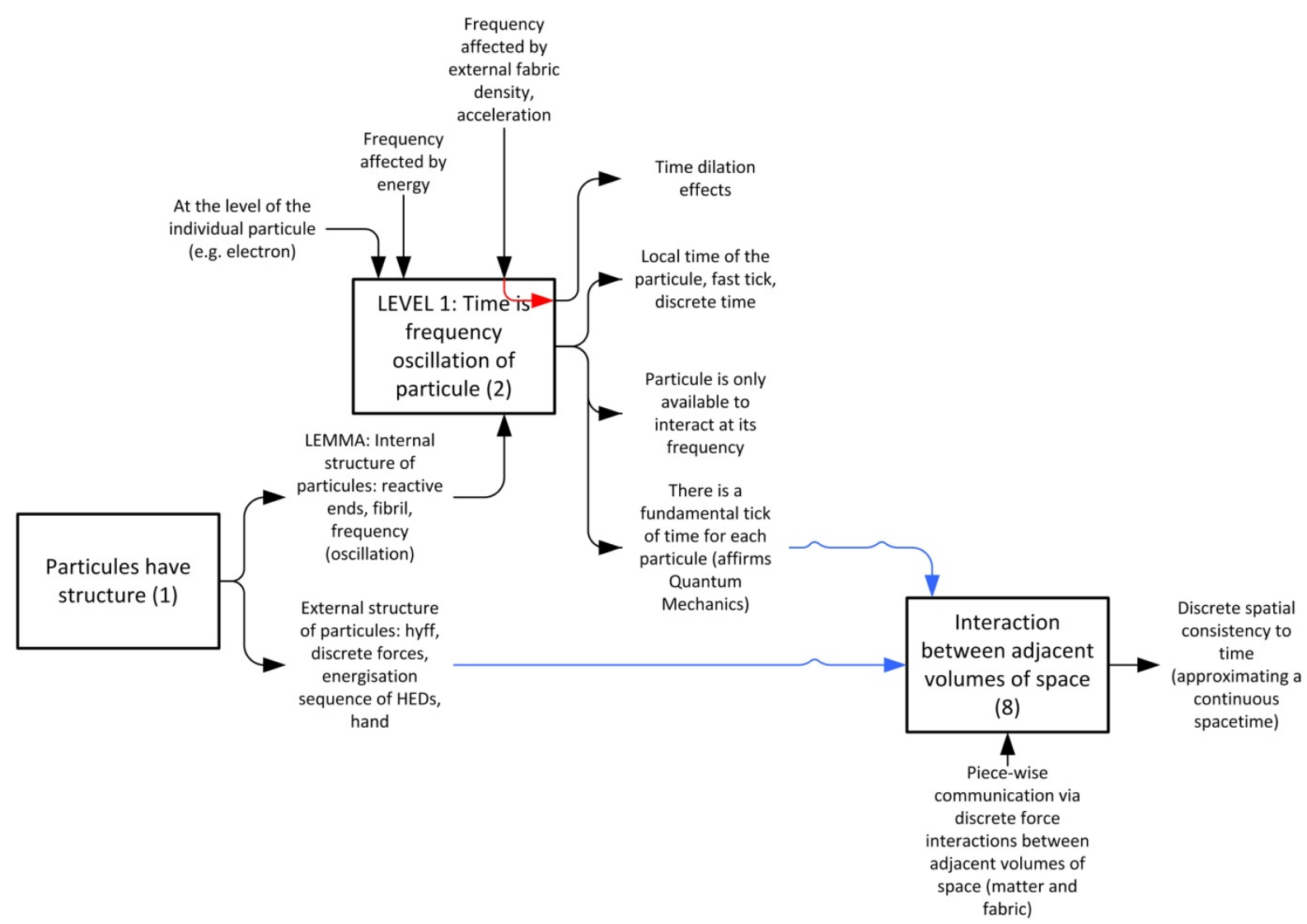

Figure 8. The apparent smoothness of time at the macroscopic level is caused by the interaction between adjacent volumes of space, mediated by discrete fields

\section{Discussion}

\subsection{Integrative Theory}

According to the Cordus theory, the fundamental time occurs with particules, being its frequency. This is the fastest time, but it also varies with particules. The way particules are assembled determines what frequency the assembly adopts - invariably lower - and how quickly it can interact with other bodies. Thus time at the macroscopic level depends on the nature of assembly of matter. Irreversibility and the arrow of time appear at the 
level where coherence fails. Human perception of time arises as a cognitive effect on top of the chemical irreversibility of physiology. Thus there is more than one time. See Figure 9 for a functional summary of the Cordus theory of time, showing all the proposed levels.

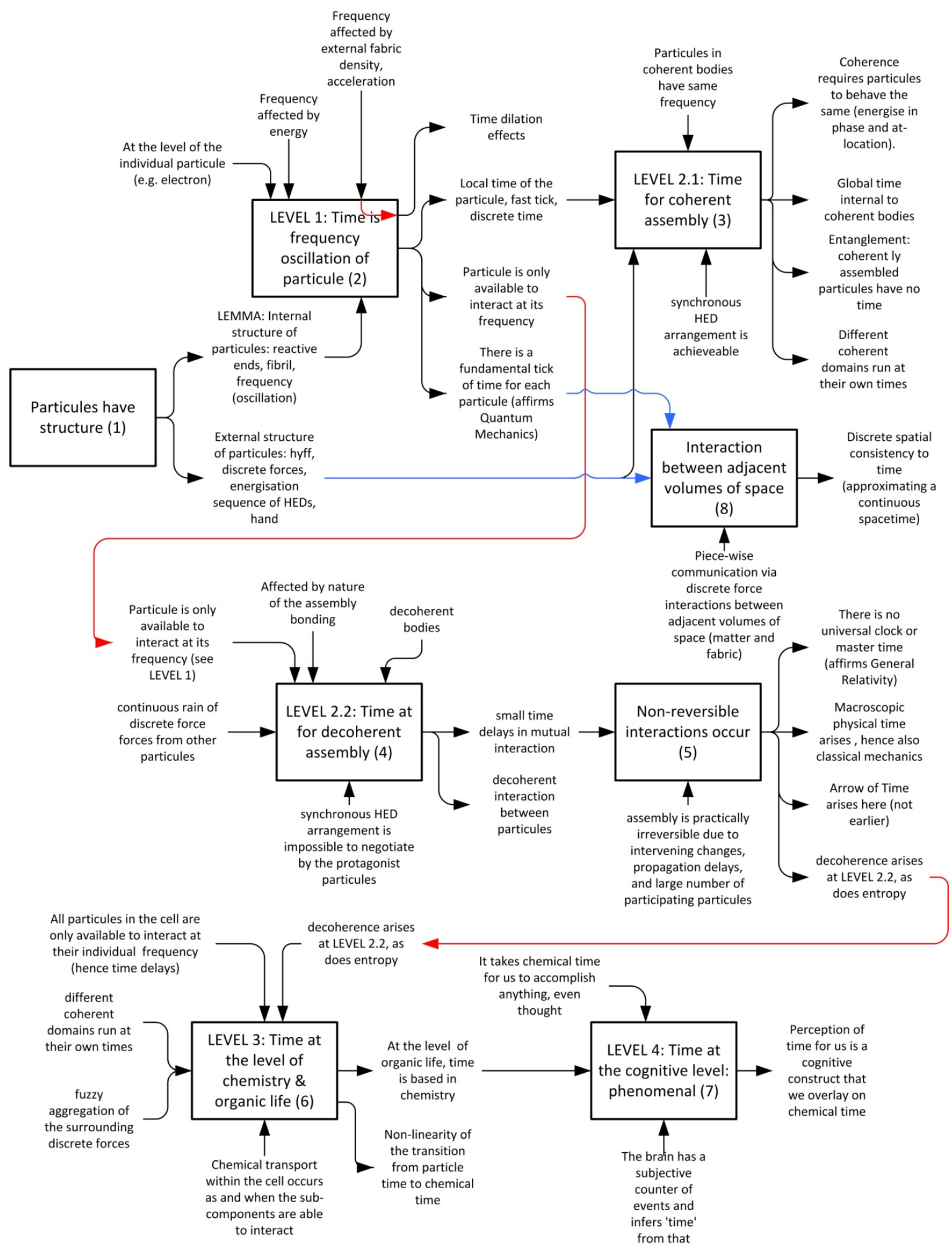

Figure 9. The Cordus functional theory for the different levels of time, and the interaction between the levels

If the Cordus theory is true, then it implies there is a deeper physics that subsumes quantum mechanics and relativity. While existing approaches have tended to focus on extending QM by the addition of discrete fields, 
through virtual bosons, these approaches have not yet been successful in solving the many ontological questions those same solutions raise. Orthodox physics relies almost exclusively on mathematical methodologies, and is also generally opposed to the hidden-variable argument. So applying design thinking is a foreign idea, and starting the search in the NLHV area perhaps seems like foolishness. Nonetheless we have shown that the design methodology can yield thought-provoking candidate solutions, and that at least one NLHV solution is not precluded by the Bell-type inequalities. The Cordus theory developed here has greater explanatory power, and a wider-ranging logical consistency than quantum mechanics or relativity.

\subsection{Outcomes}

What we have achieved here is a description of how time arises, within the Cordus framework. This work makes several novel intellectual contributions to the physics of time.

The first is the concept that time is fundamentally the frequency cycle of the particule of matter. The implication of this is that time is an emergent property of matter, is not a dimension, is not continuous, and there is no master clock or universal time. The general relativity concept of $3+1$ spacetime is denied in principle, though accepted as a practical approximation. The idea that particles have frequency is not new, being known for matter as the de Broglie frequency, and for photons as wavelength, colour and energy. However Cordus makes the novel conceptual contribution of providing a NLHV solution with a physical concept for frequency, as opposed to QM's treatment of it as an abstract "intrinsic" variable, and then linking frequency to time. Furthermore, this shows that the hidden variable sector can support a comprehensive and logically consistent theory for time.

The second contribution is the novel idea that time depends on the level of assembly of matter, from the fundamental time caused by the frequency of particule re-energisation, through to the time of assemblies of decoherent domains of matter, and onward to time at the level of organic life. This is not an idea that exists in any of the other frameworks for time. The level of assembly concept has descriptive power in its ability to reconcile multiple attributes of time within one framework.

A third contribution is an explanation of where and how irreversibility, entropy, and the arrow-of-time arise. According to the Cordus theory, these all arise at the transition from coherence to decoherence. Complementary to this is an explanation for why QM predicts time-symmetry, i.e. reversibility: because this is true for simple coherent systems. What is interesting here is that there is only one arrow-of-time which scales from the thermodynamics to the human perception (as opposed to many separate arrows a some theories propose). So this is also a parsimonious solution.

The fourth contribution is a set of explanations for how the time as measured by atomic clocks transfers to the world at large. By ascribing fundamental time to the re-energisation frequency of particules, the Cordus theory provides the necessary explanatory mechanism. So the time that we humans perceive in a cognitive sense is consistent with that measured in an objective sense by atomic clocks and other instruments. This also explains how time-dilation works. The frequency of the particule is the common cause for both the rate of time as measured by clocks and the rate at which chemical process (such as thought and aging) occur. Key to this understanding are the complementary Cordus theories for the vacuum (Cordus: fabric) and force unification. This part of the theory also explains how time begun.

Fifth, a seamless connection is provided between the various physical levels of time, and the human perception of time. This solves another ontological problem concerning how, if at all, our human perception of time connects to the physics of time. Thus time starts out as a frequency property of particules, and so it is appropriate to measure time in terms of the frequency-dependent activities of individual atoms (e.g. atomic clocks). At macroscopic levels the irreversibility arises and this drives the physiological irreversibility, and ultimately a cognitive perception of time.

A sixth contribution is some answers to philosophical questions about how the perception of the NOW arises, whether time is a dimension (no), whether it is infinitely divisible (no), whether the many-worlds theory is necessary (no), and whether it is possible to conceive of an atemporal situation (yes).

The seventh contribution is the reconciliation of multiple different forms of time into one single coherent framework. This integrates the apparently conflicting nature of the different times suggested by quantum mechanics, electromagnetic theory, and relativity. Surprisingly, it is not so much that one of these theories is correct and the others wrong, but instead it is shown that they all have a piece of truth. The Cordus theory shows that time is all of particle-based vs. spacetime, relative vs. absolute, local vs. universal. However it is not simultaneously all of those, but rather depends on the level of assembly being considered. We therefore suggest that none of the existing physical theories have got time quite right: they are all merely approximations to special 
cases. Instead Cordus suggests that there is a deeper common causality to time.

The eighth contribution is not immediately apparent in this work, but is nonetheless significant. It is the large scale coherence of the Cordus theory, whereby it is possible with one framework to provide ontologically meaningful explanations to a wide variety of otherwise problematic phenomena, including wave-particle duality, entanglement, charge-parity (CP) violation, force unification, asymmetrical baryogenesis, and now time. These solutions are conjectural and unorthodox, and their validity is uncertain, yet it is the wide applicability of the underlying Cordus principle that is interesting. There are alternative explanations for all these effects, but they tend to be piecemeal and only solve one problem. It is also curious that the Cordus theory accommodates QM and relativity, except in small details and principles of interpretation. This does not prove that the Cordus view is correct, but it does suggest it is a promising direction.

\subsection{Implications}

We have shown that the Cordus conjecture can explain time, and have therefore achieved the purpose of the paper. The explanations are consistent with those of quantum mechanics, electromagnetic theory, special and general relativity. Importantly, the Cordus theory provides a mechanism whereby the interaction with the external environment causes changes to the frequency of the particule. Neither quantum mechanics nor general relativity can do that, because they lack models for internal structure and discrete fields respectively. As we have seen, the Cordus concepts of frequency, assembly of matter, and fabric are key to this new theory of time. However, the implications are unorthodox in that it suggests that quantum mechanics, electromagnetic theory, Newtonian mechanics, and relativity are subsets and approximations of a deeper causal structure in physics. It remains to be seen whether or not the Cordus conjecture is valid, but as it stands it already has greater ontological power than any of these other theories.

\section{Relativity of simultaneity}

There are interesting implications in this Cordus theory for frames of reference and the philosophical issues surrounding time. Special relativity (SR) is based on the relativity of simultaneity, that the order in time of two spatially separate events cannot be determined absolutely, but instead depends on the motion of the observer. Thus it is impossible to order two events in time if they occur in different places (hence difference frames of reference). There is no preferred inertial frame in SR. Taken to its limits this suggests that people live in different states of the universe depending on their relative velocity, hence the Rietdijk-Putnam argument and Penrose's Andromeda paradox (Penrose, 1989). (Note 10)

The Cordus theories of time and the fabric affirm SR's principle of the relativity of simultaneity, that time can flow at different speeds for people in different situations. However there are some deeper implications from the Cordus perspective. The first is that, due to light-speed being dependent on fabric-density, there is an additional complexity to the endeavour to synchronise multiple clocks that are spatially separated (Einstein, 1920). Cordus suggests that the only reliable way to synchronise clocks is not by transmitting light signals between them, but by bringing them together into the same place at the same time, synchronising them there, and then sending them away again.

The second implication is that time is not an inherent property of space. Cordus rejects the idea of spacetime having a substantial dimensional status comparable to the three geometric axes, and instead sees the fabric as being the discrete force relationships between bodies. Complementary to this is a third implication, that time is a property of matter rather than space. Recall that the Cordus theory is that the fundamental level of time is the frequency oscillation of the particule, and the assembly of multiple particules.

This has the further implication that each assembly of matter has its own time (SR: frame of reference) which via the fabric blends discretely into that of other neighbouring matter bodies. Hence the connectedness of the Cordus fabric, which provides a mechanism whereby spatially separated bodies appraise each other about their position and state. This corresponds loosely to the GR concept of a smooth spacetime, except that the Cordus fabric is made up of discrete field elements that only appear to be smooth at the macroscopic level. A fifth implication is that spatially separate bodies have their own time, and Cordus provides a mechanism whereby that fundamental time aggregates into the physical behaviour of a clock. So the question of how time, as measured by say an atomic clock or mechanical timepiece connects to the underlying time, is answered.

This leads to another implication of the Cordus theory, which is that all the separate bodies in the universe, hence also clocks and frames of reference, were once synchronised in the past. The primary synchronisation was at the genesis of matter, when matter was formed from photons. There is a Cordus explanation for this asymmetrical baryogenesis too (Pons, D. J., Pons, A. D., \& Pons, A. J., 2011). As this matter separated in the formation of the 
universe, so it carried its clocks with it. Thus there is a branching of times (SR: frames of reference), and this also means they can all be traced back in a family tree. Therefore Cordus only conditionally supports the SP principle of relativity of simultaneity. Cordus suggests that there is a temporal relationship between different frames of reference, that the time for each body (collection of particules) represents its cumulative journey through past space and time (i.e. world-line) and that all frames can therefore be referenced back to the primal genesis event. Not that mere inspection of the matter in any one frame reveals that journey, only the sum thereof. So Cordus suggests that the temporal relationships between inertial frames of reference are not really arbitrary, but rather unapparent. Thus the relationship between two inertial clocks is not simply a convention, though it can be for convenience if the observer is willing to accept the differences as a calibration offset. While the two separate inertial clocks may each have their own time, it is generally not possible to see what this is, so the simultaneity can in practice be set by the observer's choice. So Cordus rejects the conventionality of simultaneity in principle, but allows it in practice.

\subsection{Addressing Common Questions about Time}

Time is a puzzle to researchers and the public alike. In this section we address the implications for practitioners, which in this case are other researchers and interested members of the public. Before we start, we wish to point out that the Cordus theory should be considered a conceptual solution and extended thought-experiment rather than a proven law. With that limitation in mind, here are some implications if it were to be true.

What about time travel? Can bodies travel faster than the speed of light and could this result in time flowing backward? Could spacetime be folded back on itself in a loop?

Probably no to the first. The speed of light $c$ is the local speed at which discrete forces are propagated. It is not certain that a body would be able to withstand the self-inflicted onslaught of the fabric pressure were it to travel faster than $c$, but even if this were possible its interactions with other matter would still require frequency cycles, hence time, for both participants. Even when the interactions are reversible (which is expected to only apply to the simplest levels and even then conditionally, see above), all this means is that there is no arrow of time. In every macroscopic situation there is irreversibility, hence a forward arrow of time.

Regarding the second, the folding of spacetime is not possible, according to the Cordus perspective. This is because there is no spacetime. Macroscopic time, in the Cordus theory is not a dimension at all, but a patchwork of temporal ratchets at the most fundamental level of matter. Time is a series of delayed interactions between matter, not a linear scale that can be traversed in both directions. It is not sensible, in this theory, to talk of folding time back on itself. We acknowledge that superfluids do show quantum vortices, which the Cordus theory explains as a coherent material looped back on itself, but in that case it is possible to have a void in the middle of the vortex, whereas the patchwork of time is perfused with the fabric which cannot be voided. It is not possible to connect two regions where time flows differently, because the fabric flows through both. The fabric cannot be bent, nor can time. This means that Cordus also refutes the QM idea that tiny wormholes make shortcuts through spacetime. Entanglement and the superluminal transport of information is not time travel, and is readily explainable with the Cordus theory. Nor is there any need in the Cordus theory for chronology protection (the paradox of a time-traveller killing his grandfather), because time only flows in one direction.

Is time a real fundamental property of the universe?

Yes, it is a physical effect at the particule level, the mechanism being the frequency of the particule. Yes, it is fundamental in that the existence of matter, specifically the energisation of the reactive ends, is linked to time. No, there is no master clock or universal parameter. No, in that time does not exist on its own. It is not a dimension linked to space but rather a behaviour of matter.

Is time the framework in which events take place?

No, not at least in the sense of a continuous spacetime. Yes, in that individual particules negotiate their timing (frequency, energisation) with other neighbouring particules and the fabric at large. The assembly of matter, specifically its fields, and the patchwork of negotiated interaction is the framework of time. All events occur in that framework, because all events involve interactions between particules.

Can time pass at different rates for observers in different situations? And what about time-dilation?

Yes, time is locally determined. But the different locations are linked together by negotiated discrete forces at their boundaries. Realistically those domains are very small, and large coherent volumes, e.g. vats of superfluid, are uncommon. (Where these exist the whole volume reacts as one.) Cordus explains time-dilation as the particule's discrete forces having to interact with the fabric of the vacuum, which in these cases has elevated pressure density. The interaction changes the re-energisation behaviour and slows the frequency of the particule. 
For the particule, local time is the tick of its frequency, so time really does change when the frequency does. Therefore all the process of interaction that depend on frequency, e.g. chemical reaction with a second particule, or transport of a messenger electron/atom/molecule, or emission of a photon, or nuclear decay, will happen faster/slower relative to another frame of reference. So there is absolute time at the particule level (or coherent domain) but it only applies locally. There is no universal time. The cosmos is filled not with one time, but a patchwork of many times at many levels.

Is time an illusion?

Yes, at least in that our cognitive construct of it emerges from deeper effects, and is fuzzy, being stitched together in the mind as an apparently smooth and continuous dimension. No, in the sense that time corresponds to the frequency oscillations of matter, and these exist while matter exists.

Are there alternative realities?

If there are many worlds or parallel universes, there is every reason to expect that — by definition — they will be inaccessible to the present one, and therefore unknowable. Those are metaphysical ideas, like religion in being beyond physics, and the Cordus theory cannot confirm or disprove them. Yet the Cordus theory does show there is no need for alternative realities. Cordus refutes the QM concept of many futures (temporal superposition) and provides a theory for time in which there need be only one reality in which everything that happens simply stays happened.

Is time the passage from low to high entropy?

Not quite: entropy is a related but different effect to time. The arrow of time arises at the level where decoherence results in irreversibility in the interaction between particules. While time is the frequency ticks of particules, the irreversibility of interactions contributes to the arrow of time. The same irreversibility creates entropy. But time and entropy are not the same effect, even if they have a common cause. Irreversibility is quantified by entropy, and also drives the local ratchets for the arrow of time.

Why do the laws of physics treat the past and future the same?

This is because quantum mechanics does not include entropy, in turn because it assumes that matter is always coherent (hence reversible interactions). Cordus shows why QM's assumption is incorrect, and explains why and where coherence breaks down. Likewise classical mechanics is also symmetrical regarding time, if losses are ignored. The arrow is only applied to time when irreversibility arises.

Why does the human brain not "remember" the future?

Time is a one-way effect. There is no future that is simultaneous with the present and the past. Cordus specifically refutes the idea that an object can simultaneously be in multiple futures, i.e. temporal superposition. The Cordus theory rejects the idea that the future already exists, or that time is a dimension, and instead asserts that the future depends on how the complex and irreversible now evolves.

Which perspective of time is correct: the absolute clock of quantum mechanics or the spacetime of general relativity?

Neither, but in some ways both are adequate for their purposes. According to the Cordus theory, time at the fundamental level is created by the local frequency of oscillation of the particule, and the arrow is driven by irreversibility. Thus time is locally generated, and the Cordus theory rejects the QM idea of an absolute clock (Note 11). Also, Cordus suggests that time is a patchwork at the cosmos scale, not a continuous spacetime, thereby not accepting this feature of GR either. However the theory accepts that both QM and GR are approximately correct, at least at the level of detail that concerns them. The Cordus theory provides a more primitive mechanics for time that accommodates the thoroughly different models of QM and GR.

Where did time come from?

To the level to which the Cordus theory can penetrate, time is a consequence of the frequency oscillations of particules. Its rate is thus determined by the mass of the particule, in turn how it is assembled and from what subcomponents. In that sense even massless particules (photon, neutrino) have frequency and therefore time. However the forward arrow of time arises where coherence lets off and decoherence starts. This discontinuity in the physics of time occurs at different levels of assembly depending on temperature and homogeneity. Time therefore comes from the frequency oscillation of matter, which in turn comes from the primal photon(s) at genesis. Thus time started when the universe started.

Will time end, and when? 
Time is part of matter, and shares the same origins and fate. Therefore time as we know it will cease when the universe does. This also means that there is no time (as we know it) outside the universe. This would mean that there is no time in the void into which the universe is expanding. Likewise for a being outside the universe (God) there need be no time either.

Is time a dimension?

No, it is not a dimension: it is neither smooth nor infinitely sub-divisible. It is not a ratio variable. It only looks that way when viewed from a sufficiently high level of assembly, hence the approximations of the classical mechanics. The concept of spacetime is also an approximation. In the Cordus view, time is more like a patchwork of cause-and-effect ratchets between sub-microscopic domains.

\subsection{Implications for Further Research}

The Cordus conjecture makes specific predictions that are unorthodox and thus lend themselves to testing and falsification. For example, within this paper are specific predictions about how time flows in coherent bodies such as superfluids. It might be possible to test these predictions, and thereby test the larger conjecture.

Other research implications are that there could be yet deeper mechanics to explore. Specifically in the case of time is the question of what the mechanism might be for frequency in the particule. The Cordus theory currently explains it as dynamic energy oscillation between the field structures at the two reactive ends, but undoubtedly there is more to it than this.

\section{Conclusions}

Applying the simple Cordus conjecture yields a novel alternative conceptualisation of time. According to this conceptual theory, time originates at several levels. At its most basic level time originates with the frequency cycles of the particules of matter and photons. Specifically, the ticks of time are the frequency oscillations of particules. The Cordus theory provides a specific internal structure for particules, hence a physical explanation for frequency. The rate of time is thus determined by the mass of the particule, in turn how it is assembled and from what subcomponents. The local conditions and external environment, specifically relativistic velocity, acceleration, and high gravitation, affect the energisation process of the reactive ends. This effects the frequency of the particule, and thus the local time, hence time-dilation. Thus time is locally generated, and the theory rejects the idea of an absolute or universal clock. Time therefore comes from the frequency oscillation of matter, which in turn comes from the primal photon(s) at genesis. Thus time started when the universe started, and will end with it, too.

However the ticks of time are not the same as the arrow of time. The forward arrow is only applied to time at the level where irreversibility arises. This is where coherence lets off and decoherence starts. This discontinuity in the physics of time occurs at different levels of assembly depending on temperature and homogeneity, but is well before the macroscopic or even cellular level. The Cordus theory explains how the irreversibility arises in the time-delay that is introduced (frequency ticks required) when two bodies of matter interact. This explanation applies whether those volumes are decoherent or even independently coherent. Irreversibility arises because it is statistically impossible to return all particules in the system to their original positions and states. The fabric, which comprises the discrete field forces of all the other particules in the accessible universe, adds complexity to the interaction of even the simplest assembly of particules. Therefore entropy, irreversibility, decoherence, cause-and-effect, and the arrow of time all arise at the same point.

There is a connectedness of phenomena that are at different geometric locations, and this applies between macroscopic objects and at the small scale. A phenomenon that occurs in one volume is communicated via photons, or massy particules, or fields, to other matter around it. This communication applies positional constraints on the recipient, hence temporal cause-and-effect. It is not a master clock that accomplishes this, nor does it require continuity of spacetime. The piece-wise communication between volumes of matter (whether coherent or not) achieves the macroscopic effect of time. The Cordus theory does not accept the temporal superposition of QM, hence also refuting the alternative-realities and many-worlds idea of QM. It also refutes the GR idea of spacetime, instead suggesting that macroscopic time is a patchwork of temporal ratchets, not a continuous dimension. Hence the theory also rejects the idea of time-travel via folded spacetime, or the wormhole idea of QM.

The Cordus theory offers an answer to the question of whether the absolute clock of quantum mechanics or the spacetime of general relativity is correct. Neither is, but both are adequate approximations for their purposes. The Cordus theory provides a more basic concept of time from which QM and GR emerge as different approximations. At the level of organic life, time is based in chemistry, specifically the delay introduced by the 
irreversible interaction of molecules. It takes chemical time for us to accomplish anything, even thought. Thus human perceptions of time are a construct founded on a real physical principle of temporal causality.

Thus the Cordus conjecture delivers a new theory for time, one that accommodates the existing perspectives of QM, SR and GR, but also suggests they are approximations of a deeper reality. The Cordus time theory addresses the ontology of time at the fundamental level, explains where and how the arrow-of-time arises, and describes how time might operate at the cosmological level.

The successes with the Cordus conjecture show that non-local hidden-variable solutions have merit, despite their rejection by orthodox physics. Specifically, the wider Cordus theory shows that it is possible to envisage and design a NLHV solution that circumvents the Bell-type inequalities, and has high explanatory power across many physical phenomena. In particular, we have shown that questions about time can be answered at the next deeper level of physics, and we have given an example of what that physics might look like and its implications for time.

\section{References}

Aiello, M., Castagnino, M., \& Lombardi, O. (2008). The arrow of time: from universe time-asymmetry to local $\begin{array}{lllll}\text { irreversible processes. Foundations of } & \text { 257-292. }\end{array}$ http://dx.doi.org/10.1007/s10701-007-9202-0

Bell, J. S. (1964). On the Einstein Podolsky Rosen Paradox. Physics, 1(3), 195-200. Retrieved from http://philoscience.unibe.ch/documents/TexteHS10/bell1964epr.pdf

Campisi, M., \& Hanggi, P. (2011). Fluctuation, Dissipation and the arrow of time. Entropy, 13(12), 2024-2035. http://dx.doi.org/10.3390/e13122024

Caticha, A. (2011). Entropic dynamics, time and quantum theory. Journal of Physics A: Mathematical and Theoretical, 44(22), 225303. http://dx.doi.org/10.1088/1751-8113/44/22/225303

Einstein, A. (1920). Relativity: The special and general theory. New York: Holt.

Einstein, A., Podolsky, B., \& Rosen, N. (1935). Can quantum-mechanical description of physical reality be considered complete? Physical Review, 47(10), 777. http://link.aps.org/doi/10.1103/PhysRev.47.777

FIPS. (1993). Integration definition for function modeling. (IDEF0) Retrieved 12 Aug 2003, from http://www.itl.nist.gov/fipspubs/idef02.doc

Hasker, W. (2011). Divine knowledge and human freedom. In R. Kane (Ed.), The Oxford Handbook of Free Will (pp. 39-54): Oxford University Press. http://dx.doi.org/10.1093/oxfordhb/9780195399691.003.0002

Jejjala, V., Kavic, M., Minic, D., \& Chia-Hsiung, T. (2012). Modeling time's arrow. Entropy, 14(4), 614-629. http://dx.doi.org/10.3390/e14040614

Leggett, A. (2003). Nonlocal hidden-variable theories and quantum mechanics: An incompatibility theorem. Foundations of Physics, 33(10), 1469-1493. http://dx.doi.org/10.1023/a:1026096313729

Lyre, H. (2008). Time in philosophy of physics: The central issues. Physics and Philosophy, 2008, 1-23. http://hdl.handle.net/2003/25146

Mainzer, K. (2010). The emergence of temporal structures in dynamical system. Foundations of Physics, 40(9-10), 1638-1650. http://dx.doi.org/10.1007/s10701-010-9451-1

Penrose, R. (1979). Singularities and time-asymmetry. In S. W. Hawking \& W. Israel (Eds.), General Relativity: An Einstein Centenary Survey (pp. 581-638). Cambridge University Press.

Penrose, R. (1989). The emperor's new mind. Oxford University Press.

Pons, D. J. (2011a). Stability and decay: Mechanisms for stability and initiators of decay in the neutron. viXra, 1112.0002, 1-17. Retrieved from http://vixra.org/pdf/1112.0002v1.pdf

Pons, D. J. (2012a). Limits of Coherence: Where and Why is the Transition to Discoherence? viXra, 1201.0043, 1-12. Retrieved from http://vixra.org/abs/1201.0043

Pons, D. J., \& Pons, A. D. (2012). Circumventing Bell's theorem? Design of a non-local hidden-variable model with physical substructures. viXra, 1203.0086, 1-19. Retrieved from http://vixra.org/abs/1203.0086

Pons, D. J., Pons, A. D., \& Pons, A. J. (2011). The preponderance of matter: Asymmetrical genesis via the antineutrino route. $v i X r a, 1111.0035,1-19$. Retrieved from http://vixra.org/pdf/1111.0035v1.pdf

Pons, D. J., Pons, A. D., \& Pons, A. J. (2013). Synchronous interlocking of discrete forces: Strong force 
reconceptualised in a NLHV solution. Applied Physics Research, 5(5), 107-126. http://dx.doi.org/10.5539/apr.v5n5107

Pons, D. J., Pons, A. D., Pons, A. M., \& Pons, A. J. (2011b). Electromagnetism. (Cordus in extremis Part 4.1) viXra, 1104.0027, 1-17. Retrieved from http://vixra.org/pdf/1104.0027v1.pdf

Pons, D. J., Pons, A. D., Pons, A. M., \& Pons, A. J. (2011c). Fabric of the universe. (Cordus in extremis Part 4.2) viXra, 1104.0028, 1-8. Retrieved from http://vixra.org/pdf/1104.0028v1.pdf

Pons, D. J., Pons, A. D., Pons, A. M., \& Pons, A. J. (2011d). Frequency. (Cordus optics Part 2.1) viXra, 1104.0019, 1-10. Retrieved from http://vixra.org/pdf/1104.0019v1.pdf

Pons, D. J., Pons, A. D., Pons, A. M., \& Pons, A. J. (2011e). Gravitation, Mass and Time. (Cordus in extremis Part 4.3) viXra, 1104.0029, 1-14. Retrieved from http://vixra.org/pdf/1104.0029v1.pdf

Pons, D. J., Pons, A. D., Pons, A. M., \& Pons, A. J. (2011f). Special states of matter. (Cordus matter Part 3.4) viXra, 1104.0025, 1-12. Retrieved from http://vixra.org/pdf/1104.0025v1.pdf

Pons, D. J., Pons, A. D., Pons, A. M., \& Pons, A. J. (2012b). Wave-particle duality: A conceptual solution from $\begin{array}{llll}\text { the } \quad \text { cordus } & \text { conjecture. }\end{array}$ http://physicsessays.org/doi/abs/10.4006/0836-1398-25.1.132

\section{A Appendix: Clarification of Coherence, Decoherence, and Entropy in the Cordus Theory}

\section{What is coherence?}

The Cordus theory permits a more specific definition of coherence and superposition than is possible from within the 0D point construct of QM. From the Cordus perspective, superposition is simply that the cordus particule is actually physically oscillating between two positions: the geometric locations of the reactive ends at the end of their span. The cordus particle (e.g. photon cordus) collapses to one of these ends when it is grounded. Likewise coherence, from the Cordus perspective, is when all the particules, which may be photons, electrons, protons, and possibly atoms \& molecules, etc., have synchronised frequencies and phases thereof. This also involves the sharing of hyff emission directions (HEDs).

According to this theory, coherence is a special state of assembly where the particules provide for mutual preservation of the twin locations of each others' reactive end: when any one particule is energised at its one reactive end, the position of its other dormant reactive end is filled by the active end of another particule. Thus coherence is best understood as an ordered complementary frequency state synchronisation (CoFS) between two or more particules.

For materials with a coherent structure, the effect of an externally imposed change is communicated to neighbouring internal components at the next frequency cycle. For assemblies with high purity, this may be fast indeed, hence second sound in superfluids, and rapid electron transmission across biological molecules. (Note 12) Hence also the successes in putting molecules into geometric superposition. Thus communication within atoms and molecules is rapid, being able to take advantage of the internal frequency network. However the Cordus theory does not accept temporal superposition, and hence also rejects the idea that a body can simultaneously be in multiple futures.

According to the Cordus theory, entanglement is only possible within coherent systems.

\section{What is decoherence?}

The Cordus theory anticipates three mechanisms for decoherence. First, a coherent material cannot accept internal shear velocity. Second, higher temperatures lead to decoherence because phonons (internal thermal vibrations) disturb the stability. Third, more complex assemblies of matter are harder to put into coherence, and the complicating factors are the number of components in the assembly, and the variety of species (simplicity and purity).

\section{What is entropy?}

The Cordus theory explains entropy as a spatial and temporal dilution of energy. Thus an atom that has surplus energy can dispense it in five main forms: electron orbital change (including bonding), electron ejection, photon ejection, electron flow (plasmons), and phonon propagation. If phonons, then another atom some distance away receive some of the energy and will likewise use what it can and dispense with the rest. That remote atom might emit a photon for example. Even if that photon was sent straight back to the original atom (which is not generally the case), there would still be less energy in the feedback loop because of the phonon dilution in the bulk, and the time required for the photon flight. Thus the individual mechanisms are all reversible (elastic), but the system as 
a whole is not, and we suggest this is what creates entropy.

Both photons and phonons tend to be dispersed out into the surrounding space or material (respectively), and this dilution of the original energy is the primary mechanism for thermodynamic irreversibility and entropy. The geometric and micro-structural complexity of the matter accessible to the photons and phonons introduces so many dilution paths that it is extremely unlikely that the energy fragments will spontaneously recombine. Geometric separation is another contributory factor: when the matter separates or radiates photons across space, then the dilution is further increased and the number of paths reduced by which the energy can come back together. The enormous radiative loss of photons from stars contributes to entropy, because that energy cannot realistically all be recovered after it has travelled billions of years and stopped in our eye, and even if it were reflected back it would be more billions of years to travel back. In the meantime space expands, which adds to the delay. Thus the expansion of space in the universe further contributes to entropy.

Geometric separation of matter causes the photon travelling between them to arrive late, the more so if it involves transmission through denser material. Thus the energy is not delivered at the time it might have been, but is instead postponed into the future. If that postponement is indefinite, it takes energy out of the system. This is another barrier to recombining the original energy, and thus another contribution to entropy.

Not only is the energy delayed, but so too is any information carried by the photon. Furthermore, the Cordus theory for transmission of discrete field force-elements suggests that these too travel at the speed of light. Thus information about the strength and direction of the fields of the remote particule only arrives at the basal particule after some time. The basal particule cannot respond to external fields until it receives them. This contributes a delay to the exchange of information between decoherent objects.

A critical evaluation of quantum mechanics from the perspective of the Cordus theory, suggests that the failing of QM is its inability to comprehend that it only applies to coherent situations. Thus QM is a not a universal physics but rather a special-case physics for coherent bodies only. One of several places where this failing is evident is QM's agonising over Schrodinger's Cat: its wondering why cats do not actually behave like that, and its dalliance with desperate metaphysical solutions (many worlds theorem), show how QM is unable to cope with decoherent matter. A complementary failing is QM's inability to explain why it does not scale up to the macroscopic level, or where its own boundaries of applicability might lie. To Cordus it is obvious why real cats do not display superposition: they are not coherent assemblies of matter, an a living cat never will be either. Also, the Cordus theory offers an explanation for QM's scaling problem, including a prediction of where the boundary lies (Pons, 2012a).

\section{B Appendix: Time Lemmas}

The following assumptions are built into or emerge from this Cordus theory, and expressed as lemmas. The lemmas represent the Cordus mechanics and are a mechanism to ensure logical consistency within the theory.

\section{E.4 Time Lemma}

E.4.1 Time depends on the level at which the assembly of matter occurs, being fastest for individual particules, and slowest for more complex assemblies (later identified as decoherent bodies bonded by electric-magneto-gravitational-bonding forces).

E.4.2 Fundamental Time, at the level of the individual particule (e.g. electron), is the frequency of the re-energisation cycles of its two reactive ends.

E.4.3 Fabric time is the mutual interconnectedness of matter particules spread over three-dimensional space. This occurs via the fabric, comprising discrete field forces for electric-magneto-gravitational interaction. Not strictly a time, this is rather a coordination of events across space.

E.4.4 Coherent assembly time is the common frequency cycle of re-energisation of the particules within a coherent domain of matter.

E.4.5 Decoherent assembly time is the tick of interactions within an aggregate body of coherent and decoherent sub-bodies. This tick of time is much slower than the fundamental time, and derives from individual particules responding as they can to the rain of field forces.

E.4.6 Macroscopic bodies invariably have decoherent relationships between them. Such bodies, regardless of whether they are internally governed by coherent or decoherent relationships (bonds), interact inelastically with their environment, in that such bodies do not return to precisely their initial states. Inability for one body to return thereby means that all the other bodies in the accessible universe cannot either, because the fabric of background field discrete forces has been changed. 
E.4.7 Decoherent assembly time is irreversible, hence the arrow of time arises at this level. This is because the interaction between subassemblies is practically irreversible due to intervening changes, propagation delays, and the complexity of large number of participating particules. This is also where and why entropy arises.

E.4.8 Organic Life Time, at the level of an individual cell, consists of the fuzzy aggregation of the discrete fields of the many individual coherent particules (electrons, atoms, molecules) and decoherent sub-components (clumps of molecules, organelles). "Fuzzy" because the discrete fields are not individually distinct. Chemical transport within the cell occurs as and when the sub-components are able to interact. Thus the cell takes much longer to achieve anything (more frequency ticks) than a simple sum of the times required by the coherent subcomponents.

E.4.9 Cognitive time, or the human perception of time, is a cognitive construct of the order in which we experience phenomena, based in turn on a neural representation of chemical time, and the arrow of time (irreversibility), both of which arise at a deeper level. The coordination provided by the fabric ensures that there is a consistency of experience between two people, hence personal interaction is a real common experience, and time is perceived as universal.

E.4.10 It is not a master clock that accomplishes the temporal connectedness of phenomena that are at different geometric locations, nor does it require continuity of spacetime per se. The piece-wise communication, via discrete field interactions of the fabric, between adjacent volumes of space (matter and fabric) applies spatial consistency to time. There is no universal time. The cosmos is filled not with one time, but a patchwork of many times.

\section{Notes}

Note 1. Predestination and free-will: Typical questions that arise here are: If God exists, how is it that he knows the future but yet humans still have free-will? Or are those assumptions wrong? Does God know only the present and the past state of the universe? Does God operate timelessly?

Note 2. The Zeno paradoxes: These explore the divisibility of motion. For example, in the race between the Achilles and the tortoise, where the tortoise is given an initial lead, in any one durationless moment Achilles moves closer to the tortoise, but the tortoise also moves forward a smaller amount, so by this line of thinking Achilles can never overtake the tortoise. But we do not see this, so consequently this durationless concept of time is presumably wrong as a foundation for understanding velocity. The other common modern application of Zeno's point-like analysis of effects is the quantum Zeno effect. Here a quantum system, one that is inherently randomly variable, will not change at all when under continuous observation. This taps directly into the question of the observer's role in quantum systems, whether an observer is necessary to collapse the indeterminacy of the wavefunction, and if so how. From the Cordus perspective this is straightforward to answer: contextual measurement arises because the act of measurement necessarily involves the observer using fields or matter, either of which produce discrete forces that interact with the field elements of the system under observation. Thus there is a coupling, mediated by discrete fields, and it is the nature and strength of the constraints created in this connection that results in the observer affecting (to various degrees of intrusiveness) the system being measured. The quantum Zeno effect is therefore explained in the Cordus conjecture as a delicate system being forced to behave in a certain way due to the imposition of external discrete fields that originate with the observer's measuring equipment. Thus also the observer's choice of when to make the measurement, and how to make it, affects the system under consideration. This explanation is also proposed for the delayed-choice double-slit experiment.

Note 3. Interaction determined by clocking frequency: This Cordus concept of interaction at the clocking frequency arises because the reactive end, of which each particule has two, only does anything like move or exert force, when it is active. When it is de-energised it is non-reactive. The faster the clocking frequency, the more interactions the particule can make. If this concept seems strange, it may be helpful to consider the analogy of a digital computer, which also performs interactions more intensely when provided with a clock of higher frequency.

Note 4. How does force arise? 'Force' is not quite the right word to use at this level, since the Cordus concept suggests that the mechanism is geometric displacement. The force interaction is proposed to be a synchronisation effect: the incoming discrete fields cause the recipient reactive end to energise slightly closer (or further as the case may be) than it would otherwise. Thus there is a prescribed geometric constraint on the location of re-energisation, i.e. displacement, and this Cordus suggests, is what we perceive as force at our macroscopic level. To this way of thinking the EMG field forces are therefore the strong interaction writ remotely and more loosely, since they are all HED synchronisation effects. Bonding is a medium range application of the same 
mechanism, and the strong force within the nucleus is a close-range application.

Note 5. Domain compatibility: The two domains would need to have the same frequency (hence mass characteristics) for the individual discrete forces to be apparent, in which case they could move into a bonded state of assembly, i.e. become one coherent body. Thus there is no problem with independent coherent domains merging to form larger domains, but it requires homogeneity of composition (to satisfy the mass and frequency requirements). Note that the emitted discrete forces propagate indefinitely out into the universe at light speed. They are not consumed by their interaction with other bodies -hence gravitation cannot be shielded- and cannot be recalled. So the simplest change of position of one electron has a profoundly irreversible effect on the rest of the universe. The ordered regime only applies within coherent assemblies. This tendency to irreversibility applies in several situations: in the interaction between a coherent body and the universe at large; in the interaction between multiple independent but individually coherent bodies; in the interaction of decoherent matter with the universe or other matter. The more numerous the participating domains (coherent masses or decoherent particules) the greater the tendency towards irreversibility. So the universe is practically irreversible even if the individual physical interactions are deterministic.

Note 6. The Cordus fabric: This is not made up of particles, and therefore is not a matter aether, but it is a transmission medium nonetheless, since the photon travels in the fabric. The Cordus conjecture thus affirms electromagnetic (EM) theory and its concept of a medium. Cordus also provides physical interpretations for the electric and magnetic constants of free space, which are otherwise ontologically challenging to EM theory. We also note that the Cordus fabric is a relativistic one, yet the theory suggests that the speed of light need is not constant but instead depends on the fabric density.

Note 7. Gravitational time dilation: Electric and magnetic field density does not affect time in the same way as gravitation, and the Cordus theory suggests this is because the time phenomenon is primarily connected to the mass, hence energisation sequence of the three HEDs rather than the behaviour of a single HED as in the electrostatic and magnetic interactions.

Note 8. Subjective counter of events: Exactly what 'events' the brain counts to infer passage of time is a wider mystery, and Cordus does not specifically address this cognitive question. Nor does it explain what the biological mechanism might be for accumulating the sense of elapsed time. If 'events' include external stimuli and internal markers (perhaps physiological depletion) then there is no particular difficulty explaining why perception of time is so flexible. However, a cognitive model is beyond the present scope.

Note 9. Free-will: That begs the question of whether or not there is free-will. We see some interesting ideas in there, especially given the concept of the fabric contributing to a 'unique present', plus the Cordus rejection of the many-worlds/multiverse/temporal superposition concepts (rejection not in the sense that we can disprove them but rather than we can demonstrate that we do not need them to form a coherent theory of physics). An obvious initial answer to the free-will question is that it follows the theory for time: that there is no free-will within coherent bodies, but that irredeemable irreversibility grants indeterminism to complex decoherent bodies. We hope to explore these concepts in a future paper.

Note 10. Andromeda paradox: Whether this is even a paradox is doubtful, because the two people, A and B, in Penrose's Andromeda thought experiment cannot know, at the time of their meeting, what events are transpiring far away in their difference versions of the universe. So what happens in a far galaxy like Andromeda is unknowable to the people at the time, and any difference in perception of remote events (whether or not the aliens have decided to invade Earth) is likewise unknowable. Therefore there is no contradiction in world-lines at the time of the meeting, and hence no paradox. It only appears to be a paradox to us because in a thought experiment we can 'know' what our subjects themselves do not, which is what the aliens are up to. Penrose ponders whether, at the time his subjects were meeting, there was any real uncertainty about that future, i.e. a predetermination type of question. But that assumes that A was already in a version of the universe that was more temporally advanced than B, which is not the case. He was only in version of the universe that would differ in simultaneity from B, if he had continued with his motion relative to B. Such velocity would distance A from B, and any communication from A to B would then be limited by light-speed. Or to look at it the other way, person A had not at the time of meeting $B$ been able to realise the potential advantage of foreknowledge that his temporal advancement might seem to promise. Nor would he ever be able to do so, since the information on which that knowledge was based (i.e. what decision the aliens had made) could only reach him at light-speed, by which time both he and his acquaintance B would both have moved forward in time (moved on the Minkowski diagram). Nor does B have any ability to change the unfolding decisions of the aliens, because to do so would require sending a signal back to Andromeda which would also take too long (it would only be received by the 
aliens after they had already made their decision). So while A might appear to have the benefit of advanced information about the alien's plans, that advantage cannot be realised, and therefore that information cannot be transferred to B either, and B has no real ability to communicate that information into her retarded version of the universe, and therefore has no ability to cause her retarded universe to diverse from that inhabited by A. Therefore she cannot tamper with her retarded version of the universe to change the future. There is only one world-line for the universe as a whole. Therefore what is decided by the aliens stays decided, and to answer Penrose, there is no uncertainty about the future. The future of both people is not already 'fixed', instead it is simply indeterminate. The situation only looks like a paradox to an omniscient external observer (i.e. ourselves). Thus another way to dismiss the paradox is to point out it employs circular logic: it requires its observer to be in a highly preferred reference frame, free of the relativity of simultaneity, which of course is not-permitted by special relativity.

Note 11. Contrasts between $Q M$ and Cordus: If the wave-functions of QM were rewritten in terms of the de Broglie frequency for the particule, rather than probability in absolute time, then QM and Cordus might be closer A secondary effect is that Cordus also suggests that the simple presence of an observer does not collapse the wave-function or influence the outcome of an experiment, unless that observer was bonded in a coherent way to the experiment -which Cordus suggests is practically impossible to achieve- or imposes fields/forces on the system being measured.

Note 12. For a descriptive overview of quantum biology, and applications to odour reception, electron transfer in ATP, \& photosynthesis, see Brooks, M., The weirdness inside us. New Scientist, 2011. 2832(1 October 2011): p. 34-37.

\section{Copyrights}

Copyright for this article is retained by the author(s), with first publication rights granted to the journal.

This is an open-access article distributed under the terms and conditions of the Creative Commons Attribution license (http://creativecommons.org/licenses/by/3.0/). 